\title{
Effect of dietary oils and conjugated linoleic acid on the growth performance of broilers vaccinated with the La Sota Newcastle vaccine
}

\author{
R. Aydin \\ Department of Animal Sciences, Kahramanmaras Sutcu Imam University, 46060 Kahramanmaras, Turkey
}

\begin{abstract}
The objective of this study was to investigate the effects of dietary oils on growth performance of broiler chicks vaccinated with La Sota vaccine against Newcastle disease. One hundred and seventy five 1-week old Ross PM3 male broiler chicks were randomly allocated to five dietary groups $(\mathrm{n}=35$ chicks/group) and fed diets supplemented with 0.5\% sunflower oil (Group A), 0.5\% olive oil (Group B), $0.5 \%$ beef tallow (Group C), $0.5 \%$ conjugated linoleic acid (CLA, Group D) or $0.5 \%$ hazelnut oil (Group E) for five weeks. Diets contained $230 \mathrm{~g}$ crude protein $/ \mathrm{kg}$ and $12.97 \mathrm{MJ}$ metabolisable energy (ME) $/ \mathrm{kg}$ (starter diet); $220 \mathrm{~g}$ crude protein $/ \mathrm{kg}$ and $12.97 \mathrm{MJ} \mathrm{ME} / \mathrm{kg}$ (grower diet) or 200 crude protein $/ \mathrm{kg}$ and $12.97 \mathrm{MJ}$ $\mathrm{ME} / \mathrm{kg}$ (finisher diet). The broiler chicks were vaccinated with La Sota Newcastle vaccine at 22 days of age. Body weights were measured weekly and carcass characteristics and some organ (liver, heart, gizzard and intestine) weights were determined after slaughter. The growth rates of broilers from the Groups $\mathrm{A}, \mathrm{C}$ and $\mathrm{E}$ were negatively influenced by vaccination, compared to Group $\mathrm{D}$, which maintained its growth rate. There was no difference in the proportions of abdominal fat (\%) of the chickens among the groups. The respective relative organ weights did not differ significantly between the respective dietary treatments. This study showed that the broiler chicks fed a diet supplemented with CLA had significantly better performance and carcass weights compared to the other groups. The present study also indicated that CLA included at a level of $0.5 \%$ in the diet prevented weight loss due to vaccination.
\end{abstract}

Keywords: Conjugated linoleic acid, vaccination, broiler, immune system

${ }^{\#}$ E-mail: rahimaydin@ksu.edu.tr

\section{Introduction}

Specific fatty acids such as omega-3 fatty acids and conjugated linoleic acid (CLA) were shown to improve performance and to decrease indices of inflammatory response in the growing chicks (Hellerstein et al., 1989; Cook et al., 1993; Korver \& Klasing, 1997). Omega-3 fatty acids from fish oil were shown to enhance the antibody response of chicks to sheep red blood cells, but suppressed rates of lymphocytes proliferation after mitogen stimulation (Fritsche et al., 1991). Conjugated linoleic acid is a naturally occurring substance in dairy products and meat from ruminant animals, as a result of bacterial biohydrogenation in the rumen (Ha et al., 1989) and was reported to exert a variety of biological properties in several animal models, including anti-carcinogenic (Belury, 2002), anti-atherogenic (Lee et al., 1994) and anti-diabetic (Houseknecht et al., 1998) properties. Dietary CLA was first shown to be effective in the prevention of growth depression induced by immune stimulation in chicks and mice (Cook et al., 1993). Growth suppression induced by the injection of endotoxin was markedly reduced in mice and chickens fed diets supplemented with CLA (Cook et al., 1993). Conjugated linoleic acid also promotes immunoglobulin production and modulates the production of various cytokines in rat and mouse splenocytes (Sugano et al., 1998; Yamasaki et al., 2003). Studies by Yamasaki et al. (2003) showed that the t-10, c-12 CLA isomer, but not the c-9, t-11 CLA isomer, was an active isomer to enhance immunoglobulin (Ig) production in vivo. Studies involving fish oil and CLA illustrated possible means of modulating the growth suppressive effects resulting from immune induced alterations in the nutrient metabolism (Klasing et al., 1987; Cook et al., 1993).

The immune system in poultry, like that of humans, has developed several levels of defence strategies to cope with a wide spectrum of pathogens, including parasites, bacteria and viruses (Erf, 2004). Newcastle disease is a highly contagious disease of poultry and other bird species caused by specified viruses of the avian paramyxovirus Type I serotype of the genus Avulavirus, belonging to the family of Paramyxoviridae (Mayo, 2002). Newcastle disease causes great losses in poultry in production systems where preventive 
measures are not taken. In the poultry industry vaccination against Newcastle disease with live vaccines is a common practice and even obligatory in many countries throughout the world. Stimulation of the chicken's immune system by a wide variety of immunogens decreases the rate of weight gain, feed intake and the efficiency of feed utilization (Klasing et al., 1987). Vaccination is reported to be a potent immune stimulant which depresses growth performance in poultry (Chamblee et al., 1992) and causes a decrease in the rate of protein synthesis and accretion in the skeletal muscle (Hentges et al., 1984). Oral vaccination of chicks against Newcastle disease and infectious bronchitis resulted in decreased protein synthesis and protein accretion in the skeletal muscle (Hentges et al., 1984).

In light of these findings, this study investigated the effect of supplementing broiler chick diets with CLA and different oils, and the effect thereof on the growth performance of male broiler chicks vaccinated with La Sota Newcastle vaccine.

\section{Materials and Methods}

Four hundred 1-day old Ross PM3 broiler chicks were obtained from a commercial hatchery and were housed in a room with 24-hour constant fluorescent lighting. They were vaccinated against Newcastle disease (HB1) in the hatchery. At the end of one week of feeding, sexes of the broiler chicks were determined. In this study, male broiler chicks were used because uniformity of birds is really important in an experiment. As known, body weights of the male and female birds are pretty similar at the beginning of the hatch. However, there would be a great difference in the body weights between male and female chicks at the end of 6-weeks feeding. For this reason, only male chicks were used to get better uniformity in the study. One hundred and seventy five 1-week old Ross PM3 male chicks were randomly allocated to five dietary groups $(\mathrm{n}=35)$ and fed a commercially prepared diet supplemented with $0.5 \%$ sunflower oil (Group A), $0.5 \%$ olive oil (Group B), $0.5 \%$ beef tallow (Group C), $0.5 \%$ CLA (Group D) or $0.5 \%$ hazelnut oil (Group E) for five weeks. The objective of the study was to determine the effect of different oils containing markedly different fatty acids and CLA on growth performance of the broilers vaccinated with Newcastle La Sota vaccine. Individual fatty acids have different properties due to the number and configuration of double bonds in the chain. In the current study the oils chosen represent different groups of fatty acids. For example, sunflower oil contains $69 \%$ linoleic acid; olive oil contains about $72 \%$ oleic acid and beef tallow contains higher levels of saturated fatty acids (mainly C14:0, C16:0 and C18:0). The source of CLA-80 consisted of $35.33 \% \mathrm{c}-9$, t-11 and t-9, c-11-CLA, 35.72\% t-10, c-12-CLA; $1.11 \%$ c-9, c-11-CLA; $1.57 \%$ c-10, c-12-CLA; $0.91 \% \mathrm{t}, \mathrm{t}-9,11$ and 10,12 -CLA. Other fatty acids in CLA were $6.64 \%$ palmitate, $2.39 \%$ stearate, $13.77 \%$ oleate, and $0.81 \%$ linoleate, and $1.75 \%$ unknown. Oils and CLA were mixed into the diets later.

Table 1 Chemical composition of the $\operatorname{diets}^{1}$ commercially prepared (as fed basis)

\begin{tabular}{lccc}
\hline & Starter Diet & Grower Diet & Finisher Diet \\
\hline Dry matter, g/kg & 880 & 880 & 880 \\
Crude protein, g/kg & 230 & 220 & 200 \\
Metabolic energy, MJ/kg & 12.97 & 12.97 & 12.97 \\
Ash, g/kg & 80 & 80 & 80 \\
NaCL, g/kg & 3.5 & 3.5 & 3.5 \\
Calcium, g/kg & 15 & 15 & 15 \\
Phosphorus, g/kg & 7 & 7 & 6.5 \\
Lysine, g/kg & 12 & 11 & 10 \\
Methionine, g/kg & 5 & 5 & 4 \\
Cysteine, g/kg & 4 & 4 & 3
\end{tabular}

${ }^{1}$ Also contained per kilogram of diet: $8000 \mathrm{IU}$ vitamin A; $800 \mathrm{IU}$ vitamin D3; $15 \mathrm{mg}$ vitamin E; 2 mg vitamin $\mathrm{K}_{3}$; $4 \mathrm{mg}$ vitamin $\mathrm{B}_{2} ; 10 \mathrm{mg}$ vitamin $\mathrm{B}_{12} ; 60 \mathrm{mg}$ manganese; $40 \mathrm{mg}$ zinc 
Diets were isoenergetic and isonitrogenous. The birds were allowed ad libitum access to feed and water and maintained on a 24-hour constant lighting program. When chicks reached an age of 22 days, water was withdrawn for two hours before vaccination to allow them to get thirsty. Broiler chicks were vaccinated with La Sota vaccine (Intervet International B.V. Boxmeer, Holland) reconstituted with unchlorinated water. Ample water space was provided so that all birds could get water easily. Body weights of broiler chicks were recorded weekly and mortality in the groups was recorded daily. At the end of the study the chickens were slaughtered and carcass weight and the weights of some organs (liver, heart, gizzard and intestine) were recorded. In this study, data on broiler performance and organ weights were analyzed using the general linear models procedure and differences were considered significant at a level of 95\% (SPSS software 10.0; Chicago, IL). The significance of the differences among the groups has been determined by Duncan's range test (SPSS software 10.0; Chicago, IL).

\section{Results}

Table 2 represents the effect of dietary oils and CLA on the performance of the broiler chickens vaccinated against Newcastle disease. There was no significant difference between the body weights in the groups until vaccination. However, after vaccination on day 22 after hatching, body weight performances were significantly influenced in almost all the groups, with the exception of Group D. Group D received the diet supplemented with $0.5 \%$ CLA, which prevented $(\mathrm{P}<0.05)$ the vaccine induced decrease in performance.

Table 2 The effects of dietary treatments on body weight recorded on a weekly basis in male broiler chickens

\begin{tabular}{llllll}
\hline \multicolumn{5}{c}{ Dietary treatments $^{1}$} \\
\hline Week & Group A & Group B & Group C & Group D & Group E \\
\hline 1 & $191.5 \pm 2.4$ & $186.5 \pm 3.3$ & $195.4 \pm 3.7$ & $192.9 \pm 2.7$ & $189.9 \pm 3.1$ \\
2 & $500.6 \pm 8.6$ & $507.5 \pm 9.2$ & $513.3 \pm 9.4$ & $516.2 \pm 7.7$ & $499.1 \pm 10.2$ \\
3 & $1025.2 \pm 23.3$ & $1066.5 \pm 17.2$ & $1051.3 \pm 20.4$ & $1072.2 \pm 22.3$ & $1028.2 \pm 25.3$ \\
4 & $1520.5^{\mathrm{b}} \pm 31.9$ & $1575.2^{\mathrm{ab}} \pm 25.6$ & $1566.2^{\mathrm{ab}} \pm 29.2$ & $1608.9^{\mathrm{a}} \pm 31.4$ & $1516.6^{\mathrm{b}} \pm 36.1$ \\
5 & $2068.2^{\mathrm{b}} \pm 43.9$ & $2128.1^{\mathrm{ab}} \pm 27.7$ & $2073.2^{\mathrm{b}} \pm 33.7$ & $2163.3^{\mathrm{a}} \pm 40.4$ & $2037.8^{\mathrm{b}} \pm 45.6$
\end{tabular}

${ }^{1}$ Dietary treatments: Commercial diets supplemented with $0.5 \%$ sunflower oil (Group A); $0.5 \%$ olive oil (Group B); $0.5 \%$ beef tallow (Group C); $0.5 \%$ CLA (Group D) or $0.5 \%$ hazelnut oil

${ }^{a, b}$ Means within a row lacking a common superscript differ $(\mathrm{P}<0.05)$

Table 3 shows the effect of the different dietary oils on body performance, feed conversion ratio (FCR) and carcass characteristics in male broiler chickens. Final body weights of broilers from the Groups $\mathrm{A}, \mathrm{C}$ and $\mathrm{E}$ were significantly $(\mathrm{P}<0.05)$ lower than those of the CLA-fed broilers. Dietary CLA at an inclusion level of $0.5 \%$ reduced $(\mathrm{P}<0.05)$ body weight loss. The differences in FCR were not significantly different. Eviscerated carcass weights of Group D birds were higher $(\mathrm{P}<0.05)$ compared to the carcass weights of the other three groups. The proportions of body parts and abdominal adipose tissue did not differ among the groups.

The effects of dietary treatments on the percentage of organ weights of male broilers are presented in the Table 4. No significant differences in the proportions of liver, heart and intestines of the male broilers were observed among the groups. However, the proportion of the gizzard was found to be higher $(\mathrm{P}<0.05)$ for males from Group C. 
Table 3 The effects of dietary treatments on the performance and body parts of male broilers

\begin{tabular}{llllll}
\hline & \multicolumn{5}{c}{ Dietary treatments } \\
\hline & Group A & Group B & Group C & Group D & Group E \\
\hline Initial B.W., g & $191.5 \pm 2.4$ & $186.5 \pm 3.3$ & $195.4 \pm 3.7$ & $192.9 \pm 2.7$ & $189.9 \pm 3.1$ \\
Final BW, g & $2068.2^{\mathrm{b}} \pm 43.9$ & $2128^{\mathrm{ab}} \pm 27.7$ & $2073.2^{\mathrm{b}} \pm 33.7$ & $2163.3^{\mathrm{a}} \pm 40.4$ & $2037.8^{\mathrm{b}} \pm 45.6$ \\
FCR $^{2}$ & 1.80 & 1.77 & 1.78 & 1.76 & 1.77 \\
Eviscerated carcass $^{3}$, g & $1548.6^{\mathrm{b}} \pm 38.3$ & $1574.1^{\mathrm{b}} \pm 33.8$ & $1531.5^{\mathrm{b}} \pm 33.9$ & $1648.2^{\mathrm{a}} \pm 26.8$ & $1509.3^{\mathrm{b}} \pm 40.6$ \\
Neck (\%) & $4.68 \pm 0.2$ & $4.65 \pm 0.2$ & $5.17 \pm 0.2$ & $5.04 \pm 0.1$ & $5.05 \pm 0.1$ \\
Breast $^{4}(\%)$ & $32.41 \pm 0.5$ & $33.75 \pm 0.5$ & $32.43 \pm 0.5$ & $33.24 \pm 0.5$ & $33.26 \pm 0.8$ \\
Leg $^{4}(\%)$ & $28.97 \pm 0.4$ & $27.99 \pm 0.3$ & $29.15 \pm 0.3$ & $28.55 \pm 0.3$ & $28.19 \pm 0.3$ \\
Wing $^{4}(\%)$ & $10.71 \pm 0.2$ & $10.49 \pm 0.2$ & $10.76 \pm 0.2$ & $10.26 \pm 0.2$ & $10.76 \pm 0.1$ \\
Back (\%) & $23.24 \pm 0.3$ & $23.11 \pm 0.2$ & $22.49 \pm 0.4$ & $22.92 \pm 0.4$ & $22.75 \pm 0.3$ \\
Abdominal fat (\%) & $1.36 \pm 0.09$ & $1.53 \pm 0.06$ & $1.46 \pm 0.13$ & $1.42 \pm 0.11$ & $1.56 \pm 0.08$
\end{tabular}

${ }^{\mathrm{T}}$ Commercial diets supplemented with $0.5 \%$ sunflower oil (Group A); $0.5 \%$ olive oil (Group B); $0.5 \%$ beef tallow (Group C); 0.5\% CLA (Group D) or 0.5\% hazelnut oil

${ }^{2} \mathrm{FCR}=$ feed intake / body weight gain

${ }^{3}$ Eviscerated carcass $=$ carcass without head, internal organs, abdominal fat and feet

${ }^{4}$ Percentage of eviscerated carcass

${ }^{\mathrm{a}, \mathrm{b}}$ Means within a row lacking a common superscript differ $(\mathrm{P}<0.05)$

Table $4{ }^{1}$ Percentage of organ weights of male broilers fed diets supplemented with different oils for 5 weeks

\begin{tabular}{|c|c|c|c|c|c|}
\hline \multicolumn{6}{|c|}{ Dietary treatments ${ }^{2}$} \\
\hline & Group A & Group B & Group C & Group D & Group E \\
\hline Liver $(\%)$ & $2.70 \pm 0.09$ & $2.55 \pm 0.07$ & $2.60 \pm 0.07$ & $2.52 \pm 0.13$ & $2.57 \pm 0.06$ \\
\hline Heart $(\%)$ & $0.81 \pm 0.04$ & $0.79 \pm 0.03$ & $0.83 \pm 0.04$ & $0.79 \pm 0.02$ & $0.79 \pm 0.03$ \\
\hline Gizzard (\%) & $1.87^{b} \pm 0.06$ & $1.84^{\mathrm{b}} \pm 0.07$ & $2.17^{\mathrm{a}} \pm 0.11$ & $1.82^{b} \pm 0.07$ & $1.87^{b} \pm 0.06$ \\
\hline Intestine (\%) & $5.81 \pm 0.34$ & $5.56 \pm 0.18$ & $5.61 \pm 0.20$ & $5.48 \pm 0.10$ & $5.50 \pm 0.16$ \\
\hline
\end{tabular}

\section{Discussion}

The possibilities of immune stimulants in growing poultry chicks are numerous and could induce antigen exposure from cuts, management procedures (toe and beak trimming) and stimulants in the air, water and feed (Cook et al., 1993). One important immune stimulant was reported to be vaccination procedure against disease outbreaks (Chamblee et al., 1992). As vaccination against the deadly Newcastle virus prevents mortality, weight loss in chickens is being neglected in the poultry industry. Vaccination of chicks was reported to cause a decrease in the rate of protein synthesis in the skeletal muscle and protein accretion (Hentges et al., 1984). Klasing et al. (1987) also reported that stimulation of the immune system by a wide variety of immunogens decreased feed intake, feed conversion rate and weight gain in growing chicks. 
CLA was shown to act as a growth factor in rats (Chin et al., 1994) and pigs (Ostrowska et al., 1999). In a study conducted in male broiler chickens, the influence of dietary CLA on early inflammatory responses induced by lipopolysaccharide was studied and found that supplementation of CLA into the diets partially prevented reductions in body weight gain (Takahashi et al., 2002). Another study conducted in laying hens showed that dietary CLA modulated certain aspects of the immune system (Politis et al., 2003). The authors suggested that the increased quantity of urokinase plasminogen activator on the membrane of macrophages and heterophils isolated from hens fed the CLA diet might facilitate the ability of those cells to reach the point of a potential inflammation (pro-inflammatory effect) (Politis et al., 2003). In the present study broilers fed a diet containing $0.5 \%$ CLA prevented $(\mathrm{P}<0.05)$ vaccine-induced reductions in the body weights. Following immune stimulation, cytokines of interleukin-1 (IL-1) and tumour necrosis factor (TNF- $\alpha$ ) released by macrophages induce degradation of skeletal muscle and decrease muscle synthesis (Klasing et al., 1987). It was reported that IL-1 stimulated the production of muscle prostaglandin $\mathrm{E}_{2}\left(\mathrm{PGE}_{2}\right)$ (Hellerstein et al., 1989) which is an elongated desaturated product of linoleic acid. Interleukin-1 is a cytokine produced by macrophages following immune stimulation and is capable of decreasing rate of gain and feed intake (Klasing et al., 1987). In a study conducted in rats, it was shown that rats fed a diet containing fish oil did not exhibit a characteristic depression in feed intake when injected with IL-1 (Hellerstein et al., 1989). Application of $\mathrm{PGE}_{2}$ directly into muscle also caused a wasting process (Rodemann \& Goldberg, 1982). Two eicosanoids important in the inflammatory response are PGE series and leukotrienes of the B series (Korver \& Klasing, 1997). After feeding diets containing 0\% (control), 0.5\% or $1 \%$ CLA for three weeks, there was a trend toward a reduction in the release of leukotriene B4 (LTB4) from the exudate cells in response to the dietary CLA levels (Sugano et al., 1998).

Studies involving fish oil and CLA illustrated possible means of modulating the growth suppressive effects resulting from immune induced alterations in the nutrient metabolism. Omega- 3 fatty acids are generally known to decrease the levels of pro-inflammatory cytokines. i.e. IL-1, IL-6 and TNF- $\alpha$ and increase the levels of anti-inflammatory cytokines e.g. IL-2 (Chandrasekar \& Fernandes, 1994). In a study conducted on mice, it was reported that CLA supplementation resulted in increased T-cell proliferation and enhanced IL-2 by splenocytes (Hayek et al., 1999). Previously, dietary CLA was reported to decrease the production of prostaglandins in a number of tissues (Cunningham et al., 1997; Whigham et al., 1998). Another study conducted in early weaning pigs suggested that dietary CLA enhances cellular immunity by modulating phenotype and effector functions of $\mathrm{CD} 8^{+}$cells involved in both adaptive and innate immunity (Bassaganya-Riera et al., 2001). It was reported that t-10, c-12 CLA, but not c-9, t-11 CLA, was an active isomer of CLA to enhance immunoglobulin (Ig) production in vivo (Yamasaki et al., 2003). (Sugano et al., 1998) found that splenic levels of IgA, IgG, and IgM increased while those of IgE decreased significantly in rats fed 1\% CLA diet.

In the present study, dietary oils except CLA did not prevent vaccine-induced loss in the body weights. Broiler chickens fed a diet supplemented at the level of $0.5 \%$ gained significantly $(\mathrm{P}<0.05)$ more in body weight than the other groups. This may be due to prevention of the catabolic activity of immune stimulation by feeding CLA in the broilers (Cook et al., 1993). Zhang et al. (2005) showed that dietary CLA at the level of $1 \%$ elevated antibody production in male broiler chickens. In the present study, feeding $0.5 \%$ hazelnut oil (high in C18:3, n-3) was not effective to prevent vaccine-induced body weight loss. Cook et al. (1993) found that growth suppression induced by the injection of endotoxin was markedly reduced in mice and chickens fed diets supplemented with CLA. CLA, which is a group of positional and geometrical isomers of linoleic acid (C18:2, n-6), was found to be more effective to alleviate the effects of endotoxin injection on the growth rate compared to the fish oil. In a study of Miller et al. (1994) it was shown that mice fed a basal diet or diet with $0.5 \%$ fish oil lost twice as much in body weight after endotoxin injection than mice fed CLA. Cook et al. (1993) hypothesized that dietary CLA protected against cytokine-induced muscle wasting by altering the eicosanoid metabolism pathway.

\section{Conclusions}

The growth rate in the broilers fed diets supplemented with $0.5 \%$ sunflower oil or hazelnut oil was negatively influenced after vaccination, when compared to the other treatment groups. The relative organ weights of the broilers did not differ significantly between dietary treatments. This study showed that the broiler chicks fed a diet supplemented with CLA had better $(\mathrm{P}<0.05)$ performance and carcass weights. The 
present study also indicated that dietary CLA at a level of $0.5 \%$ protected weight loss due to vaccination against Newcastle disease. The actual mechanism of how dietary CLA modulates the effects of vaccination or any antigen is not fully understood. This study might help the poultry industry to prevent the adverse effects of vaccination against Newcastle disease.

\section{References}

Bassaganya-Riera, J., Hontecillas, R., Zimmerman, D.R. \& Wannemuehler, M., 2001. Dietary conjugated linoleic acid modulates phenotype and effector functions of porcine $\mathrm{CD} 8^{+}$lymphocytes. J. Nutr. 131, 2370-2377.

Belury, M.A., 2002. Inhibition of carcinogenesis by conjugated linoleic acid: Potential mechanisms of action. J. Nutr. 132, 2995-2998.

Chamblee, T.N., Thompson, J.R. \& Thaxton, J.P., 1992. Effect of day old vaccination on broiler performance. Poult. Sci. 71 (Suppl. 1), 144 (Abstr.)

Chandrasekar, B. \& Fernandes, G., 1994. Decreased pro-inflammatory cytokines and increased antioxidant enzyme gene expression by $\omega-3$ lipids in murine lupus nephritis. Biochem. Biophys. Res. Comm. 200, 893-898.

Chin, S.F., Storkson, J.M., Albright, K.J., Cook, M.E. \& Pariza, M.W., 1994. Conjugated linoleic acid is a growth factor for rats as shown by enhanced weight gain and improved feed efficiency. J. Nutr. 124, 2344-2349.

Cook, M.E., Miller, C.C., Park, Y. \& Pariza, M.W., 1993. Immune modulation by altered nutrient metabolism: nutritional control of immune-induced growth depression. Poult. Sci. 72, 1301-1305.

Cunningham, D.C., Harrison, L.Y. \& Shultz, T.D., 1997. Proliferative responses of normal human mammary and MCF-7 breast cancer cell to linoleic acid, conjugated linoleic acid and eicosanoid synthesis inhibitors in culture. Anticancer Res. 17, 197-204.

Erf, G.F., 2004. Cell-mediated immunity in poultry. Poult. Sci. 83, 580-590.

Fritsche, K.L., Cassity, N.A. \& Huang, S., 1991. Effect of dietary fat source on antibody production and lymphocyte proliferation in chickens. Poult. Sci. 70, 611-617.

Ha, Y.L., Grimm, N.K. \& Pariza, M.W., 1989. Newly recognized anticarcinogenic fatty acids: identification and quantification in natural and processed cheeses. J. Agric. Food Chem. 37, 75-81.

Hayek, M.G., Han, S.N., Wu, D., Watkins, B.A., Meydani, M., Dorsey, J.L., Smith, D.E. \& Meydani, S.N., 1999. Dietary conjugated linoleic acid influences the immune response of young and old C57BL/6CrIBR mice. J. Nutr. 129, 32-38.

Hellerstein, M.K., Meydani, S.N., Meydani, M., Wu, K. \& Dinarello, C.A., 1989. Interleukin-1-induced anorexia in the rat. Influence of prostaglandins. J. Clin. Invest. 84, 228-235.

Hentges, E.J., Marple, D.N., Roland Sr, D.A. \& Pritchett, J.F., 1984. Muscle protein synthesis and growth of two strains of chicks vaccinated for Newcastle disease and infectious bronchitis. Poult. Sci. 63, $1738-1741$.

Houseknecht, K.L., Heuvel, J.P., Vanden Moya-Camarena, S.Y., Portocarrero, C.P., Peck, L.W., Nickel, K.P. \& Belury, M.A., 1998. Dietary conjugated linoleic acid normalizes impaired glucose tolerance in the Zucker diabetic fatty fa/fa rat. Biochem. Biophys. Res. Commun. 244, 678-682.

Klasing, K.C., Laurin, D.E., Peng, R.K. \& Fry, D.M., 1987. Immunologically mediated growth depression in chicks: influence of feed intake, corticosterone and interleukin-1. J. Nutr. 117, 1629-1637.

Korver, D.R. \& Klasing, K.C., 1997. Dietary fish oil alters specific and inflammatory immune responses in chicks. J. Nutr. 127, 2039-20-46.

Lee, K.N., Kritchevsky, D. \& Pariza, M.W., 1994. Conjugated linoleic acid and atherosclerosis in rabbits. Atherosclerosis 108, 19-25.

Mayo, M.A., 2002. A summary of taxonomic changes recently approved by ICTV. Arch. Virol. 147, $1655-1656$.

Miller, C.C., Park, Y., Pariza, M.W. \& Cook, M.E., 1994. Feeding conjugated linoleic acid to animals partially overcomes catabolic response due to endotoxin injection. Biochem. Biophys. Res. Commun. 198, 1107-1112.

Ostrowska, E., Muralitharan, M., Cross, R.F., Bauman, D.E. \& Dunshea, F.R., 1999. Dietary conjugated linoleic acids increase lean tissue and decrease fat deposition in growing pigs. J. Nutr. 129, 2037-2042. 
Politis, I., Dimopoulou, M., Voudouri, A., Noikokyris, P. \& Feggeros, K., 2003. Effects of dietary conjugated linoleic acid isomers on several functional properties of macrophages and heterophils in laying hens. Br. Poult. Sci. 44, 203-210.

Rodemann, H.P. \& Goldberg, A.L., 1982. Arachidonic acid, prostaglandin $E_{2}$ and $F_{2}$ influence rates of protein turnover in skeletal and cardiac muscle. J. Biol. Chem. 257, 1632-1638.

Sugano, M., Tsujita, A., Yamasaki, M., Noguchi, M. \& Yamada, K., 1998. Conjugated linoleic acid modulates tissue levels of chemical mediators and immunoglobulins in rats. Lipids 33, 521-527.

Takahashi, K., Kawamata, K., Akiba, Y., Iwata, T. \& Kasai, M., 2002. Influence of dietary conjugated linoleic acid isomers on early inflammatory responses in male broiler chickens. Br. Poult. Sci. 43, 47-53.

Whigham, L.D., Cook, E.B., Stahl, J.L., Saban, R., Pariza, M.W. \& Cook, M.E., 1998. Conjugated linoleic acid reduced antigen-induced prostaglandin E2 release from sensitized tracheas. Fed. Am. Soc. Exp. Biol. 12, A819.

Yamasaki, M., Chujo, H., Hirao, A., Koyanagi, N., Okamoto, T., Tojo, N., Oishi, A., Iwata, T., YamauchiSato, Y., Yamamoto, T., Tsutsumi, K., Tachibana, H. \& Yamada, K., 2003. Immunoglobulin and cytokine production from spleen lymphocytes is modulated in C57BL/6J mice by dietary cis- 9 , trans11 and trans-10, cis-12 conjugated linoleic acid. J. Nutr. 133, 784-788.

Zhang, H., Guo, Y. \& Yuan, J., 2005. Conjugated linoleic acid enhanced the immune function in broiler chicks. Br. J. Nutr. 94, 746-752. 